\title{
Micro-Ikebana by Biomimetic Crystallization of Alkaline Earth Carbonates*
}

\author{
Shota Kobayashi \\ Chitose Institute of Science and Technology, Graduate School of Photonics Science, \\ Bibi 65-758, Chitose 066-8655, Hokkaido, Japan
}

\author{
Olaf Karthaus ${ }^{\dagger}$ \\ Chitose Institute of Science and Technology, Department of Applied Chemistry and Bioscience, \\ Bibi 65-758, Chitose 066-8655, Hokkaido, Japan \\ Viktor Fischer ${ }^{\ddagger}$ \\ Chitose Institute of Science and Technology, Department of Applied Chemistry and Bioscience, \\ Bibi 65-758, Chitose 066-8655, Hokkaido, Japan, and \\ Japan Society for the Promotion of Science, 5-3-1 Kojimachi, Chiyoda-ku, Tokyo 102-0083, Japan
}

(Received 9 January 2017; Accepted 25 March 2017; Published 13 May 2017)

\begin{abstract}
Biomineralization is the process of forming hard exo- or endoskeltons by biological organisms. The physical properties and the morphology of the composite material that is formed depends on the mode of crystal-growth, which depends on the diffusion of the constituents (metal cations, their anions, and additives).

We chose a model system, strontium carbonate / silica, because the precipitation of $\mathrm{SrCO}_{3}$ and $\mathrm{SiO}_{2}$ is $\mathrm{pH}$ dependent. A lower $\mathrm{pH}$ accelerates $\mathrm{SiO}_{2}$ formation while it slows down $\mathrm{SrCO}_{3}$ crystallization, and vice versa. Thus, the precipitation of the composite can occur in an alternating fashion, regulating the morphology of the carbonate precipitate. The solution of $\mathrm{SrCl}_{2}$ and water glass is poured into a shallow container and a glass slide is floated on top, eliminating the effects of bulk precipitation and convection. The $\mathrm{pH}$ is adjusted to a starting value of 12 , and a small amount of dimethyl carbonate is added. This compound slowly hydrolyses and gives off carbon dioxide, the carbonate source for the crystal growth.

The crystal morphology is clearly dendritic and shows several distinct periods of growth. First, a few crystals grow from a common seed, and after a while, the blocking of surface sites on the carbonate crystals by water glass, leads to the start of a more needle-like growth with a significant increase of branching density. Finally, after seven days, coral-like structures are formed. [DOI: 10.1380/ejssnt.2017.65]
\end{abstract}

Keywords: Electron microscopy; Crystallization; Diffusion and migration; Alkaline earth metals; Dendritic and/or fractal surfaces

\section{INTRODUCTION}

Biomineralization is the process of organisms to produce an inorganic material. For example, shellfish, corals and phytoplankton such as coccolithophorids are generally well-known. The complicated structure of the coccolithophorid shell is influenced by the environment, e.g. temperature, $\mathrm{pH}$, and $\mathrm{CO}_{2}$ concentration. Their crystal shape is radically different from abiogenic bulk crystals [1], in which the unit cells of the crystal lattice arrange in a regular three-dimensional solid body with smooth sides and edges [2]. The creation of biomimetic versions of biominerals, that are formed by mimicking some aspect of the conditions under which biominerals form, can be useful for applications that require high-surface area materials, such as catalysts [3] or column materials for separation or purification [4].

One popular approach to control the biomimetic crystal growth is the addition of polymers $[5,6]$. Such additives allow the selective growth along certain crystal planes and also allow for the stabilization of metastable crystal phases. Inorganic additives such as $\mathrm{SiO}_{2}$ can also be used [7], and we have reported on the fabrication of dendritic, flower-like, and coral-like microcrystals in a $\mathrm{SrCl}_{2}$

\footnotetext{
* This paper was presented at the 8th Asian Conference on Nanoscience and Nanotechnology (AsiaNANO 2016), Sapporo Convention Center, Sapporo, Japan, October 10-13, 2016.

$\dagger$ Corresponding author: kart@photon.chitose.ac.jp

‡ Present address: Mitsubishi Polyester GmbH, Mainz, Germany
}

\begin{abstract}
$/ \mathrm{SiO}_{2}$ system [8]. In the present paper, we further focus on the control of the crystalline form by changing the conditions of crystal growth.
\end{abstract}

\section{EXPERIMENTAL}

Strontium chloride hexahydrate (Wako, 99.0\%), sodium silicate solution (water glass; Wako $57.0 \%$, mol ratio $\mathrm{SiO}_{2} / \mathrm{Na}_{2} \mathrm{O}_{2}$ : 2.06), dimethyl carbonate (Wako, 98.0\%), and sodium hydroxide (Wako, 97.0\%) were purchased commercially and used without further purification. Distilled water was used for the preparation of all required solutions. Glass cove slides (Matsunami NEO micro cover glass, $25 \times 50 \mathrm{~mm}$, thickness $0.12-0.17 \mathrm{~mm}$ ) were used as the crystallization substrate. The substrate was intensively cleaned with acetone and 2-propanol prior to use. Crystallization experiments were conducted in disposable petri dishes with a diameter of $8.5 \mathrm{~mm}$. Stock-solutions of strontium chloride hexahydrate $(0.1 \mathrm{M})$ and sodium silicate were used as the strontium carbonate and silica precursor respectively. The sodium silicate stock-solution was prepared by mixing $10 \mathrm{~g}$ of commercial water glass with $90 \mathrm{~g}$ of water and the addition of $15 \mu \mathrm{l}$ of $\mathrm{NaOH}(1$ $\mathrm{M})$. In a typical diffusion controlled crystallization experiment an aliquot of the strontium chloride solution (200 $\mu \mathrm{l}$ for the $1 \mathrm{mM}$ experiment, $600 \mu \mathrm{l}$ for the $3 \mathrm{mM}$ experiment, and $1 \mathrm{ml}$ for the $5 \mathrm{mM}$ experiment) were mixed with water to a total volume of $20 \mathrm{ml}$, followed by the addition of $200 \mu \mathrm{l}$ of silica precursor (resulting $\mathrm{pH}=12.0$ ). 


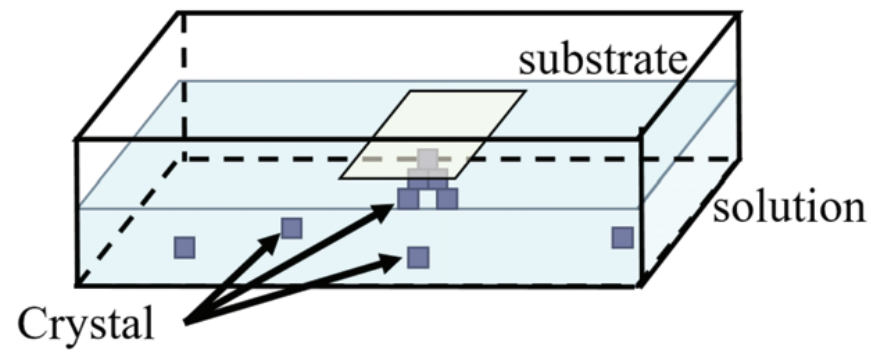

FIG. 1. Schematic diagram of experimental setup with the floating substrate.

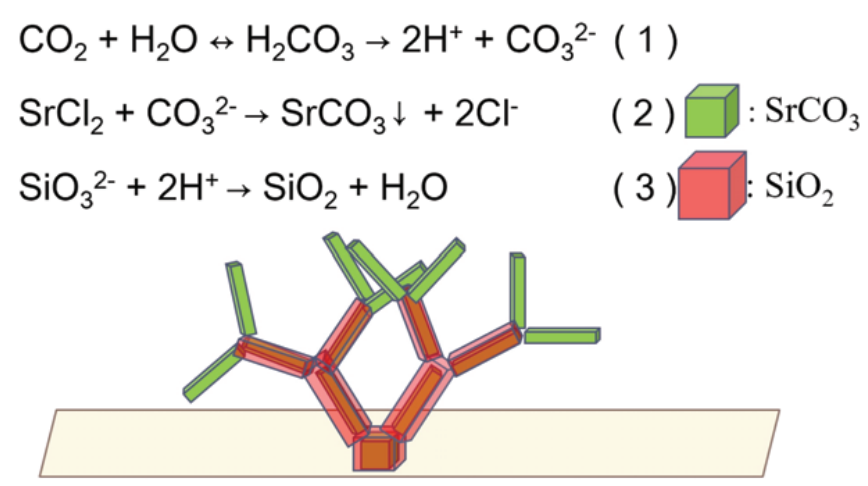

FIG. 2. Schematic diagram of crystal growth and chemical reaction formulae.

This solution was poured in polystyrene dishes with a surface area of $10 \mathrm{~cm}^{2}$. Finally, the glass substrate was gently placed at the water surface, and the crystallization setup was covered with a Büchner funnel to avoid disturbance of the sample due to air movement. After substrate was placed at the air/water interface, $100 \mu \mathrm{l}(1.19 \mathrm{mmol})$ of dimethyl carbonate were added to the crystallization medium for experiments involving DMC and covered by Parafilm@ to restrict $\mathrm{CO}_{2}$ absorption from air. According to http://camblab.info/wp/index.php/parafilmfrequently-asked-questions/, Parafilm has a $\mathrm{CO}_{2}$ permeability of $\leq 1100 \mathrm{~cm}^{3} / \mathrm{m}^{2} / 24 \mathrm{~h}$ at $23^{\circ} \mathrm{C}$ and $50 \%$ relative humidity, so that it is estimated that a maximum of 20 $\mu \mathrm{mol}$ of $\mathrm{CO}_{2}$ could have diffused into the reaction medium each day. The samples were removed from the water surface after three to seven days, and washed copious times with water and $\mathrm{EtOH}$. The sample was allowed to dry in air for further investigations with scanning electron microscopy. SEM pictures were taken with a JSM-7800F (JEOL, Japan), and element mapping was performed with an attached EDS equipment.

\section{RESULTS AND DISCUSSIONS}

Figure 1 is a schematic diagram of the experiment. W.Noorduin had immersed the substrate in the solution [7]. Crystallization from solution leads to concentration depletion, and thus less density of the solution in the vicinity of the formed crystals, and under normal gravity conditions, vertical convection sets in. Thus
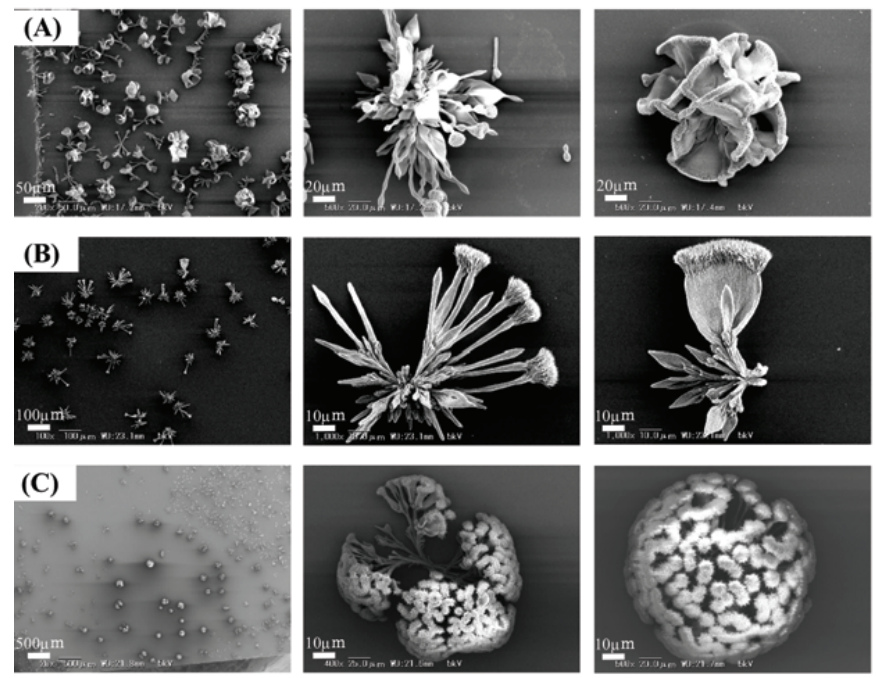

FIG. 3. SEM pictures of $\mathrm{SrCO}_{3}$ crystals, atmospheric $\mathrm{CO}_{2}$, 7days (A) $1.0 \mathrm{mM}$; (B) $3.0 \mathrm{mM}$; (C) $5.0 \mathrm{mM}$.

unevenly distributes material and leads to heterogeneous crystallization. By floating the substrate on the solution, those effects of gravity and liquid convection on the crystal growth are limited. In previous experiments, we have used atmospheric $\mathrm{CO}_{2}$ as the carbonate source. $\mathrm{CO}_{2}$ has to diffuse into the reaction solution, and because the floating substrate covers some of the water surface, the carbonate concentration under the substrate shows a gradient. In most of the experiments described here, we use dimethylcarbonate (DMC) as a carbonate source, since DMC hydrolyses in alkaline solution to release $\mathrm{CO}_{2}$ homogeneously in the solution. The strontium carbonate and silicon dioxide present in the solution precipitate alternately onto the substrate, and gradually $\mathrm{SrCO}_{3}$ crystals will grow, as can be seen in the scheme in Figure 2. The pH decreases when reactions (1) and (2) take place, because their net reaction is $\mathrm{CO}_{2}+\mathrm{H}_{2} \mathrm{O}+\mathrm{SrCl}_{2} \rightarrow \mathrm{SrCO}_{3}+2 \mathrm{HCl}$. On the other hand, the $\mathrm{pH}$ increases during reaction 3 , because protons are used up to transform silicate ions into $\mathrm{SiO}_{2}$. Thus, when reaction 1 and 2 occur, reaction (3) accelerates, depleting the solution of $\mathrm{SiO}_{3}^{2-}$ ions. Since the $\mathrm{SiO}_{2}$ precipitate on a $\mathrm{SrCO}_{3}$ crystal will block most crystallization sites, and thus will reduce further $\mathrm{H}^{+}$production, the $\mathrm{SiO}_{2}$ production will eventually become slower. The remaining active crystallization sites on the $\mathrm{SrCO}_{3}$ crystals will then again lead to increased $\mathrm{SrCO}_{3}$ precipitation, and decrease in $\mathrm{pH}$. In a non-equilibrium state in which reactants can diffuse through the solution, reaction $(1)+(2)$ and (3) will thus occur in a spatio-temporal pattern. Only selected areas on a $\mathrm{SrCO}_{3}$ crystal surface are available for crystal growth, leading to highly branched, dendritic, crystals, as is schematically depicted in Figure 2 .

The low magnification scanning electron microscopy (SEM) images in the left columns of Figs. $3-5$ give the reader a qualitative assessment on crystal density, shape, and size variation. The high magnification images in the center and right columns are representatives of the most commonly observed crystal types.

SEM images in Fig. 3 show that the morphology of the 

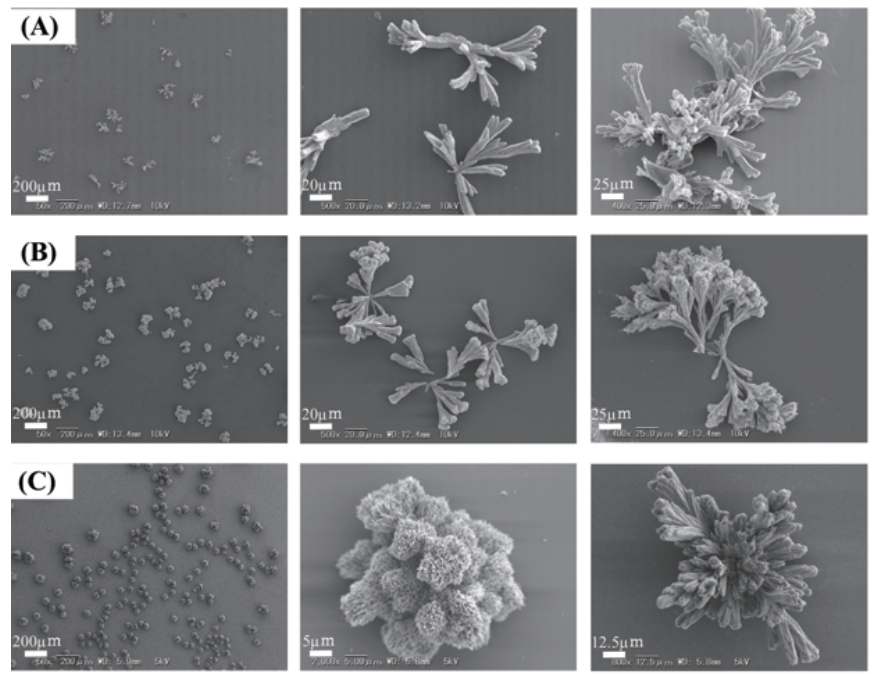

FIG. 4. SEM pictures of $\mathrm{SrCO}_{3}$ crystals with DMC, 3days (A) $1.0 \mathrm{mM}$; (B) $3.0 \mathrm{mM}$; (C) $5.0 \mathrm{mM}$.
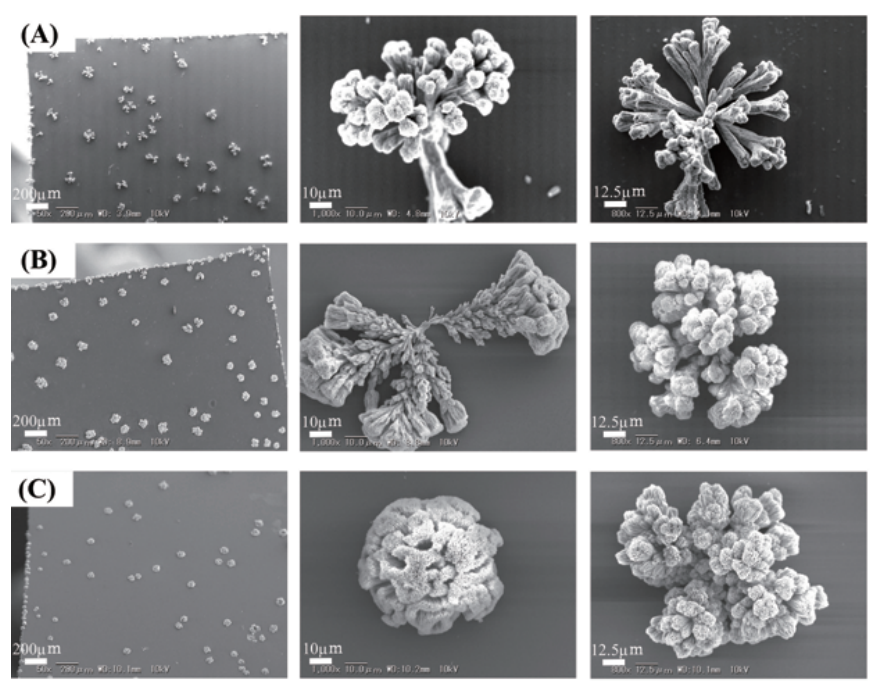

FIG. 5. SEM pictures of $\mathrm{SrCO}_{3}$ crystals with DMC, 7days (A) $1.0 \mathrm{mM}$; (B) $3.0 \mathrm{mM}$; (C) $5.0 \mathrm{mM}$.

fabricated crystals after 7 days depend on the solute concentration, especially when no DMC was added. Low concentration $\left(1 \mathrm{mM} \mathrm{SrCl}_{2}\right)$ gives leaf-like structures, while there are more flower-like structures at high concentration $\left(3 \mathrm{mM} \mathrm{SrCl}_{2}\right)$ and hemispherical shapes of crystals $\left(5 \mathrm{mM} \mathrm{SrCl}_{2}\right)$. One drawback of the $\mathrm{CO}_{2}$ diffusion technique is the insufficient control of crystal shapes. Since the $\mathrm{CO}_{2}$ concentration varies spatially (more $\mathrm{CO}_{2}$ at the edges of the floating glass slide and less under the center part of the glass slide, because the glass slide blocks the diffusion of atmospheric $\mathrm{CO}_{2}$ into the reaction solution), the crystal growth parameters are different, resulting is different crystal shapes across the glass slide.

One way to improve the homogeneity of the crystals is to employ a homogeneous $\mathrm{CO}_{2}$ concentration throughout the solution. Dimethyl carbonate hydrolyses at high pH resulting in carbonate and methanol, and thus by adding DMC to the solution, we can achieve a high and homoge-
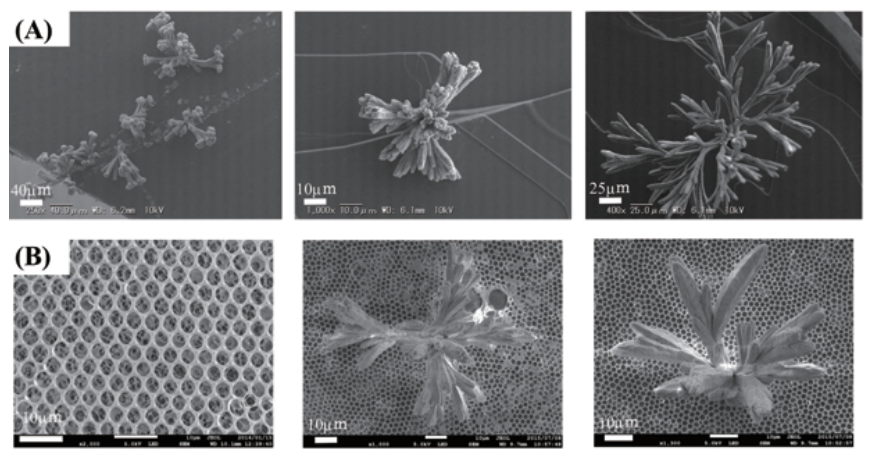

FIG. 6. SEM pictures of $\mathrm{SrCO}_{3}$ crystals with DMC, 7 days $1.0 \mathrm{mM}$ (A) mica substrate; (B) honeycomb film.
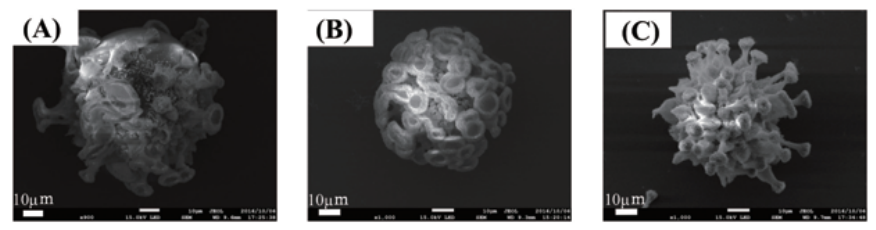

FIG. 7. SEM pictures of $\mathrm{SrCO}_{3}$ crystals with DMC, glass beads template, $1.0 \mathrm{mM} \mathrm{Sr}$.

neous $\mathrm{CO}_{2}$ concentration. Compared to the usual carbon dioxide source from air, dimethyl carbonate gave more dense structures, as can be seen in Figs. 4 and 5. The 'stems' of the flower-like structures have a larger diameter, and are less branched. Also, the 'flower-heads' are denser and less branched for all concentrations, meaning that the solubility product of $\mathrm{SrCO}_{3}$ is reached even at lower $\mathrm{Sr}$ concentrations, because of the high carbonate concentration. Between three and seven days of crystal growth, the structures become even more dense, and a broccoli-like structure is formed. Thus we conclude that the higher carbonate concentration in the aqueous solution leads to faster crystal grow, before the $\mathrm{SiO}_{2}$ blocking layer can form.

We did not carry out x-ray crystallography in order to determine the crystal structure of the formed carbonates, because it already has been reported that even large organic additives, such as p-aminobenzioc acid, or N-(2hydroxyethyl)ethylenediamine- $\mathrm{N}, \mathrm{N}, \mathrm{N}$-triacetic acid did not change the crystal structure, and always the most stable orthorhombic strontionite [8].

As we have previously reported, the crystallization takes place at random nucleation sites on the glass substrate surface [9]. In order to evaluate what types of surfaces induce crystallization, we used different types of surfaces: a very smooth, defect free hydrophilic surface of mica - an aluminium silicate-, a very rugged amphiphilic polymer honeycomb surface [10], and glass beads. Since mica is an anionic atomically flat surface with exposed silicate anions, we expected to see a similar crystallization blocking by the addition of water glass to the $\mathrm{SrCl}_{2}$ solutions. And indeed, mica did not induce crystallization or dendritic flower-like growth. Figure 6 shows that the atomically flat mica surface is free of crystals. Only a few crystals are present at the step edges that were present on 

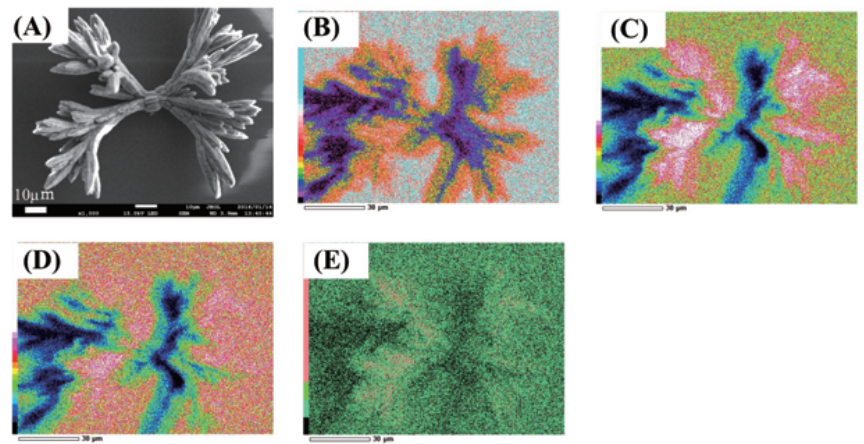

FIG. 8. EDS mapping, Sr $1.0 \mathrm{mM}, 7$ days (A) SEM image; (B) Oxygen map; (C) Strontium map; (D) Silicon map; (E) Carbon map.
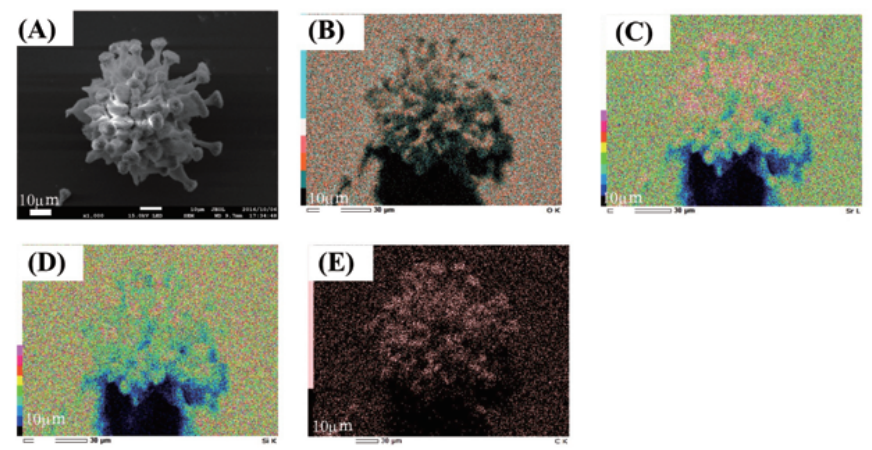

FIG. 9. EDS mapping, Sr $1.0 \mathrm{mM}, 7$ days (A) SEM image; (B) Oxygen map; (C) Strontium map; (D) Silicon map; (E) Carbon map.

the mica surface after peeling. Even though honeycomb surfaces can be used to immobilize inorganic nanoparticles $[11,12]$ and induce a patterned adsorption of metal complexes [13], the highly rugged honeycomb surface is not suitable to induce crystallization either. Figure 6 shows just a few crystals at random positions on the honeycomb surface. Glass beads, on the other hand, act similar to planar glass surfaces and induce crystal seeding and growth (Fig. 7). Compared to flat glass surfaces, it is striking that the density of the $\mathrm{SrCO}_{3}$ crystals is extremely high. The whole glass surface is covered with crystals to the extent that the glass surface cannot be seen anymore, and this is evidence for a much higher nucleation site density on a comparatively rough spherical glass surface as compared to a smooth, industrially made glass slide.

From the elemental analysis mapping with EDX in Figs. 8 and 9 , it was verified that $\mathrm{Sr}, \mathrm{C}$ and $\mathrm{O}$ were contained in the crystals that were grown both on flat substrates as well on glass micro beads, suggesting that they consist of $\mathrm{SrCO}_{3}$.

\section{CONCLUSIONS}

We found that $\mathrm{SrCO}_{3}$ dendritic crystals form when a $\mathrm{SrCl}_{2}$ solution is mixed with water glass and covered with a floating glass, mica or honeycomb substrate. The coprecipitating water glass has a strong influence on crystal growths. Furthermore, the shape and dentritic structure can be controlled by adding dimethyl carbonate as a carbon dioxide source. Such structures may be useful as solid supports for catalysts, or to study the biometic crystal growths of biogenic dendritic inorganic crystals.

\section{ACKNOWLEDGMENTS}

A part of this work was conducted in Chitose Institute of Science and Technology, supported by Nanotechnology Platform Program (Synthesis of Molecules and Materials) of the Ministry of Education, Culture, Sports, Science and Technology (MEXT), Japan.
[1] S. Weiner and P. M. Dove, Rev. Mineral. Geochem. 54, 1 (2003).

[2] A. Mersmann, Crystallization Technology Handbook (Taylor \& Francis, 2001).

[3] J. Ge, J. Lei, and R. N. Zare, Nat. Nanotechnol. 7, 428 (2012).

[4] L.-S. Zhong, J.-S. Hu, H.-P. Liang, A.-M. Cao, W.-G. Song, and L.-J. Wan, Adv. Mater. 18, 2426 (2006).

[5] S.-S. Wang, A. Picker, H. Cölfen, and A.-W. Xu, Angew. Chem. Intl. Ed. 52, 6317 (2013).

[6] F. Natalio, T. P. Corrales, M. Panthöfer, D. Schollmeyer, I. Lieberwirth, W. E. G. Müller, M. Kappel, H.-J. Butt, and W. Tremel, Science 339, 1298 (2013).
[7] W. L. Noordiun, A. Grinthal, L. Mahadevan, and J. Aizenberg, Science 340, 823 (2013).

[8] B. Sreedhar, Ch. S. Vani, D. K. Devi, V. Sreeram, and M. V. B. Rao, Am. J. Mat. Sci. 2, 142 (2012).

[9] V. Fischer and O. Karthaus, Phys. Chem. Chem. Phys. 17, 6695 (2015)

[10] O. Karthaus, N. Maruyama, X. Cieren, M. Shimomura, H. Hasegawa, and T. Hashimoto, Langmuir 16, 6071 (2000).

[11] K. Kon and O. Karthaus, Proc. SPIE 7364, 73640K (2009).

[12] T. T. Meiling, H.-G. Löhmannsröben, K. Kon, and O. Karthaus, e-J. Surf. Sci. Nanotech. 8, 309 (2010).

[13] K. Kon, C. N. Brauer, K. Hidaka, H.-G. Löhmannsröben, and O. Karthaus, Langmuir 26, 12173 (2010). 